\title{
Integrated production and routing planning decisions under uncertainty: a case study
}

\author{
Manuel Díaz-Madroñero ${ }^{1}$, David Peidro ${ }^{1}$, Josefa Mula ${ }^{1}$ \\ ${ }^{1}$ Research Centre on Production Management and Engineering (CIGIP) \\ Universitat Politècnica de València \\ Escuela Politécnica Superior de Alcoy, Plaza Ferrándiz y Carbonell, 2, 03801 Alcoy (Alicante-SPAIN) \\ fcodiama@cigip.upv.es, dapeipa@cigip.upv.es, fmula@cigip.upv.es
}

\begin{abstract}
In this paper, a fuzzy mathematical programming model is proposed to address integrated production and routing planning decisions which considers uncertain production and inventory capacities, as well as production, setup, inventory holding and transport costs. This proposal considers flexible constraints and the lack of knowledge related to the previous input parameters which are modelled by using triangular fuzzy numbers. Results of computational experiments performed with data from a case study related to a real-world bakery firm confirm the efficiency of the proposed solution method in terms of total costs and CPU time with respect to a deterministic formulation.
\end{abstract}

Keywords: production routing; uncertainty; fuzzy mathematical programming; fuzzy sets

\section{Introduction}

In today's competitive environment, organizations need to optimize their production and transport plans. Generally, these decisions have been made sequentially and independently. The most habitual procedure is, firstly, to determine production plans to meet customer demand by taking into account inventory levels and the different production costs by using lot-sizing models [1]-[3] and, secondly, to establish distribution routes and the set of products that should be sent in each vehicle by considering vehicle routing problem (VRP) models [4], [5]. However, an integrated production and transport planning may lead to increased efficiency and cost savings. In this sense, a considerable number of scientific articles on this topic have been published in recent decades, as well as several review papers [6]-[10]. Nevertheless, most of these models only consider direct shipments as a transport strategy because they disregard routing planning decisions.

In this context, production and routing models emerge in order to simultaneously plan production and distribution decisions by considering transport routes calculations. This kind of integrated production and distribution planning problem is called production and routing problem (PRP) by several authors [11], [12]. According to Bard and Nananukul (2010), in a production routing problem, four critical decisions have to be made: (1) how many items to manufacture each day; (2) when to visit each customer; (3) how much to deliver to a customer during a visit; and (4) which delivery routes to use.

The PRP is a recent research area and it has been, mainly, developed in recent years. In the seminal works by Chandra and Fisher [14] and Fumero and Vercellis [15] is shown the value of integrated production and transport routing decisions by comparing an integrated planning approach versus a decoupled one. On the other hand, Lei et al. [16] provide a PRP with multiple capacitated production plants, all of which are able to produce the same product, and many customers, each with a deterministic demand for the product over a finite planning horizon. The formidable complexity of the PRP has made most of authors have focused on developing efficient solution methods such as metaheuristics [13], [17]-[23] validated with artificially generated instances instead of case studies or realistic problems.

The complex nature and dynamics of the relationships among the different factors in industrial environments imply an important degree of uncertainty in planning decisions. According to Mula et al. [24] and Peidro et al. [25], the scientific literature provides various planning models under uncertainty. Those models defined by analytical approaches, simulation approaches or hybrid approaches (based on the integration of analytical and simulation models) represent uncertainty based on probability distributions, which are generally, based on historical data. However, a PRP operates in an uncertainty scenario in which statistical data are not very reliable, or are not even available. It can, scarcely, admit that the future values of certain parameters, like demand and capacity, have a frequentistic nature and are, therefore, likely to be treated by a stochastic approach [26]. Therefore, when statistical data are not very reliable neither available, the determination-based models of these probability distributions may not be the best option. In this context, fuzzy mathematical programming can prove to be an alternative approach to model the different types of uncertainty inherent to PRP.

According to Adulyasak et al. [27], there is no contributions which consider PRP in an uncertain environment. In this paper, we propose a fuzzy mixed integer linear programming (FMILP) model for modelling a PRP which considers uncertain costs either production and inventory capacities.

The rest of the paper is arranged as follows: Section 2 presents the PRP notation and the corresponding model formulation. Section 3 describes the solution methodology. Next, Section 4 evaluates the behavior of the proposed model in a real-world bakery firm. Final- 
ly, Section 5 provides conclusions and directions for further research.

\section{Notation and mathematical formulation}

In general terms, the production routing problem can be defined in a network $G=(N, A)$, where $N$ represents the set of nodes comprising production plant and customers, and $A$ represents the set of arcs connecting the nodes, where $A=\{(i, j): i, j \in N, i \neq j\}$ with the following notation:

\section{Sets}

$T \quad$ Set of time periods $(t=1, \ldots, T)$

$N \quad$ Set of nodes $(i=0, \ldots, N)$ and $(j=0, \ldots, N)$, where the plant is represented by node 0 and $N c=N \backslash\{0\}$ is the subset of customers

$K \quad$ Set of vehicles $(k=1, \ldots, K)$

\section{Parameters}

$d_{i t} \quad$ Demand at node $i$ during period $t$

$p \widetilde{c} \quad$ Unitary manufacturing cost

$s \widetilde{c} \quad$ Setup cost

$i \widetilde{c}_{i} \quad$ Holding cost at node $i$

$\tilde{c}_{i j} \quad$ Travel cost between nodes $i$ and $j$

$P \tilde{C}$ ap Production capacity at the manufacturing plant

$I \widetilde{C} a p_{i} \quad$ Inventory capacity at node $i$

VCap Vehicle capacity

Decision variables

$P_{t} \quad$ Production amount during period $t$

$I_{i t} \quad$ Inventory level at node $i$ at the end of period $t$

$Q_{i k t} \quad$ Amount delivered to customer $i$ with vehicle $k$ during period $t$

$\gamma_{t} \quad$ Binary setup during period $t\left(\gamma_{t}=1\right.$, if a setup is performed during period $t, 0$ otherwise)

$Y_{i t} \quad$ Binary variable equal to 1 if node $i$ is visited during period $t, 0$ otherwise

$X_{i j k t} \quad$ Binary variable equal to 1 if vehicle $k$ travels from node $i$ to node $j$ during period $t, 0$ otherwise

The PRP under uncertainty is formulated as follows:

$\min \sum_{t \in T}\left(\begin{array}{l}p \tilde{c} \cdot P_{t}+s \tilde{c} \cdot \gamma_{t}+\sum_{i \in N} i \tilde{c}_{i} \cdot I_{i t}+ \\ \sum_{i \in N} \sum_{j \in N} \sum_{k \in K} \tilde{c}_{i j} \cdot X_{i j k t}\end{array}\right)$

subject to

$$
\begin{aligned}
& I_{0 t}=I_{0, t-1}+P_{t}-\sum_{i \in N c} \sum_{k \in K} Q_{i k t} \quad \forall t \in T \\
& I_{i t}=I_{i, t-1}+\sum_{k \in K} Q_{i k t}-d_{i t} \quad \forall i \in N c, \forall t \in T \\
& P_{t} \leq M \cdot \gamma_{t} \quad \forall t \in T \\
& P_{t} \widetilde{\simeq} \boldsymbol{P} \tilde{C} a p \quad \forall t \in T \\
& I_{i t} \tilde{\leq} I \tilde{C} a p_{i} \quad \forall i \in N, \forall t \in T
\end{aligned}
$$

$$
\begin{aligned}
& Q_{i k t} \leq M \cdot \sum_{j \in N} X_{i j k t} \quad \forall i \in N c, \forall k \in K, \forall t \in T \\
& \sum_{i \in N c} Q_{i k t} \leq \text { VCap } \\
& \sum_{k \in K} \sum_{j \in N} X_{i j k t}=Y \\
& \sum_{j \in N} X_{j i k t}=\sum_{j \in N} X_{i j k t} \quad \forall i \in N c, \forall k \in K, \forall t \in T \\
& \sum_{j \in N c} X_{0 j k t} \leq 1 \\
& \forall k \in K, \forall t \in T \\
& \sum_{i \in S} \sum_{j \in S} X_{i j k t} \leq|S|-1 \forall S \subseteq N c,|S| \geq 2, \forall k \in K, \forall t \in T \\
& P_{t}, I_{i t}, Q_{i k t} \geq 0 \text { and integer } \forall i \in N, \forall k \in K, \forall t \in T \\
& \gamma_{t}, Y_{i t}, X_{i j k t} \in\{0,1\} \quad \forall i, j \in N, \forall k \in K, \forall t \in T
\end{aligned}
$$

The objective function (1) corresponds to the minimization of total costs relating to production, setups, inventories and routing costs over the planning horizon. Constraints (2) and (3) represent the inventory flow balance at the plant and customer warehouses, respectively. These equations link the production side with the routing problem through decision variables $Q_{i k t}$. The inventory of a certain product in a node, at the end of the period, will be equal to the inputs minus the outputs of the product generated in this period. Inputs concern the production (at the plant), transport receptions from other nodes (at customers) the inventory of the previous period. Outputs are related to shipments to customers (at the plant) and demand (at customers). Constraint (4) ensures that a setup is done during a period if production is taken place in this period. Constraint (5) limits the total amount to produce in the production plant during each period. Symbol $\widetilde{r}$ represents the fuzzy version of $\leq$ and means "essentially less than or similar to". This constraint shows that the planner wants to make the left-hand side of the constraint, the production amount per period, smaller or similar to the right-hand side, the maximum production capacity available, "if possible". The production capacity is only known approximately, due to, for example, machine breakdowns, and is represented by a fuzzy number. The same ocurrs with inventory capacity. Constraint (6) establishes the inventory limits of each product at each node (e.g., plant or customers). Constraints (7) to (12) deal with the typical routing equations in VRP detailed in Toth and Vigo [28]. Only if the node $i$ is visited by the vehicle $k$ during the period $t$ can the delivered an amount of product $p$ by this vehicle be positive, as stated in Constraint (7). Moreover, Constraint (8) limits the total amount of different products to be transported in a single vehicle according to its capacity. Constraint (9) ensures that the variable $Y_{i t}=1$ when the node $i$ is visited by a vehicle. Constraint (10) corresponds to vehicle conservation flow; that is, if a vehicle $k$ arrives at a customer $i$ during the period $t$, it must leave it during the same period. Constraint (11) limits to 1 the number of trips per vehicle leaving the depot for each period, and Constraint (12) eliminates possible subtours for each vehicle during each period. Constraint (13) defines the lower bounds and integrality of the production, invento- 
ry and shipment amounts. Finally, Constraint (14) defines the binary variables relating to setups, visits to customers and travelled arcs.

\section{Solution methodology}

In this section, taking into account to Peidro et al. [29], an approach to transform the FMILP model into an equivalent auxiliary crisp MILP model for a PRP planning under production and inventory capacities and costs uncertainties is defined. In order to address the fuzzy coefficients of the FMILP model, it is necessary to consider the fuzzy mathematical programming approaches that integrally consider the fuzzy coefficients of the objective function and the fuzzy constraints. Since the FMILP model considers incomplete or imprecise information in data (associated to: production costs, setup costs, inventory holding costs, transport costs, production and inventory capacity) and fuzziness associated to the flexible constraints (5) and (6), we need a fuzzy optimization approach that jointly considers the possible lack of knowledge in data and existing fuzziness. For this reason, the approach by Cadenas and Verdegay [30] is adopted in this paper. The authors propose a general model for fuzzy linear programming that considers fuzzy cost coefficients, fuzzy technological coefficients and fuzzy right-hand side terms in constraints. Fuzziness is also considered in the inequalities that define the constraints. This general fuzzy linear programming model is as follows:

$$
\begin{array}{ll}
\text { Max } & \sum_{j=1}^{n} \tilde{c}_{j} x_{j} \\
\text { s.t. } & \sum_{j=1}^{n} \tilde{a}_{i j} x_{j} \tilde{\leq} \tilde{b}_{i} \\
& x_{j} \geq 0, i \in M, j \in N
\end{array}
$$

where the fuzzy elements are given by:

- For each cost $\exists \mu_{j} \in F(\Re)$ so that $\mu_{j}: \mathfrak{R} \rightarrow[0,1], j$ $\in N$, which defines the fuzzy costs.

- For each row $\exists \mu_{i} \in F(\mathfrak{R})$ so that $\mu_{i}: \mathfrak{R} \rightarrow[0,1], i$ $\in M$, which defines the fuzzy number in the right-hand side of constraints.

- For each $i \in M$ and $j \in N \exists \mu_{i j} \in F(\Re)$ so that $\mu_{i j}: \mathfrak{R} \rightarrow[0,1]$, which defines the fuzzy number in the technological matrix.

- For each row $\exists \mu_{i} \in F[F(\Re)]$ so that $\mu_{i}$ : $F(\Re) \rightarrow[0,1], i \in M$ which provides the accomplishment degree of the fuzzy number for each $x \in \mathfrak{R}^{n}$

$$
\widetilde{a}_{i 1} x_{1}+\widetilde{a}_{i 2} x_{2}+\ldots+\widetilde{a}_{i n} x_{n}, i \in M
$$

with regard to the $i$ th constraint, that is, the adequacy between this fuzzy number and the one $\tilde{b}_{i}$ in relation to the $i$ th constraint.

A solution method based on the substitution of (15) by a convex fuzzy set through a ranking function as a comparison mechanism of fuzzy numbers is proposed by Cadenas and Verdegay [30].
Let $A, B \in F(\Re)$; a simple method for ranking fuzzy numbers consists of defining a ranking function mapping each fuzzy number into the real line, $g: F(\Re) \rightarrow \mathfrak{R}$. If this function $g(\cdot)$ is known, then:

$g(A)<g(B) \Leftrightarrow \mathrm{A}$ is less than $\mathrm{B}$

$g(A)>g(B) \Leftrightarrow \mathrm{A}$ is greater than $\mathrm{B}$

$g(A)=g(B) \Leftrightarrow \mathrm{A}$ is equal to $\mathrm{B}$

Usually, $\mathrm{g}$ is called a linear ranking function if: $\forall A, B \in F(\Re), g(A+B)=g(A)+g(B)$

$\forall r \in \mathfrak{R}, r>0, g(r A)=r g(A), \forall A \in F(\Re)$

In order to solve the problem, (15) defines: let $\mathrm{g}$ be a fuzzy number linear ranking function and given the function, $\Psi: F(\mathfrak{R}) \times F(\mathfrak{R}) \rightarrow F(\Re)$ so that:

$$
\psi\left(\widetilde{a}_{i} x, \tilde{b}_{i}\right)=\left\{\begin{array}{cc}
\tilde{t}_{i}, & \widetilde{a}_{i} x \leq_{g} \tilde{b}_{i} \\
\tilde{t}_{i}(-) \widetilde{a}_{i} x(+) \tilde{b}_{i} & \tilde{b}_{i} \leq_{g} \widetilde{a}_{i} x \leq_{g} \widetilde{b}_{i}(+) \tilde{t}_{i} \\
0, & \widetilde{a}_{i} x \leq_{g} \tilde{b}(+) \tilde{t}_{i}
\end{array}\right.
$$

Where $\tilde{t}_{i} \in F(\mathfrak{R})$ is a fuzzy number in such a way that its support is included in $\mathfrak{R}^{+}$, and $\leq_{g}$ is a relationship that measures that $A \leq_{g} B, \forall A, B \in F(\Re)$, and (-) and $(+)$ are the usual operations among fuzzy numbers.

According to Cadenas and Verdegay [30], the membership function associated with the fuzzy constraint $\tilde{a}_{\mathrm{i}}$ $\mathrm{x} \tilde{\underline{b}} \tilde{b}_{i}$, with $\tilde{t}_{i}$, a fuzzy number giving the maximum violation of the ith constraint is:

$\mu^{i}: F(\Re) \rightarrow[0,1] / \mu^{i}\left(\widetilde{a}_{i} x, \widetilde{b}_{i}\right)=\frac{g\left(\psi\left(\widetilde{a}_{i} x, \tilde{b}\right)\right)}{g\left(\widetilde{t}_{i}\right)}$

where $g$ is a linear ranking function.

Given the problem (15), $\widetilde{\Xi}$ with the membership function (26) and using the decomposition theorem [31], [32] for fuzzy sets, the following is obtained:

$$
\begin{aligned}
& \mu^{i}\left(\widetilde{a}_{i} x, \widetilde{b}_{i}\right) \geq \alpha \Leftrightarrow \frac{g\left(\psi\left(\widetilde{a}_{i} x, \tilde{b}\right)\right)}{g\left(\widetilde{t}_{i}\right)} \geq \alpha \Leftrightarrow \frac{\left.g\left(\tilde{t}_{i}(-) \tilde{a}_{i} x(+) \tilde{b}\right)\right)}{g\left(\widetilde{t}_{i}\right)} \geq \alpha \Leftrightarrow \\
& g\left(\widetilde{t}_{i}\right)-g\left(\widetilde{a}_{i} x\right)+g\left(\widetilde{b}_{i}\right) \geq g\left(\widetilde{t}_{i}\right) \alpha \Leftrightarrow g\left(\widetilde{a}_{i} x\right) \leq g\left(\widetilde{b}_{i}(+) \tilde{t}_{i}(1-\alpha)\right) \Leftrightarrow \\
& \widetilde{a}_{i} x \leq_{g} \widetilde{b}_{i}+\tilde{t}_{i}(1-\alpha)
\end{aligned}
$$

where $\leq_{g}$ is the relationship corresponding to $g$.

Therefore, an equivalent model to solve (15) is the following:

$$
\begin{aligned}
& \operatorname{Max} z=\sum_{j=1}^{n} \tilde{c}_{j} x_{j} \\
& \text { s.t. } \\
& \sum_{j=1}^{n} \tilde{a}_{i j} x_{j} \leq_{g} \tilde{b}_{i}+\tilde{t}_{i}(1-\alpha), \\
& x_{j} \geq 0, i \in M, j \in N, \alpha \in[0,1]
\end{aligned}
$$

In order to solve (17), we apply a linear ranking function, the first index of Yager [33], [34]. Although the approach could be easily adapted to the use of any other index. Thus, by applying the first index of Yager and by considering triangular fuzzy numbers, the problem defined in Eq. (15) is transformed into the crisp equivalent linear programming problem defined in Eq. (18): 
$\operatorname{Max} z=\sum_{j=1}^{n}\left(c_{j}+\frac{d_{c_{j}}-d_{c_{j}}^{\prime}}{3}\right) x_{j}$

s.t.

$\sum_{j=1}^{n}\left(a_{i j}+\frac{d_{a_{i j}}-d_{a_{i j}^{\prime}}^{\prime}}{3}\right) x_{j} \leq\left(b_{i}+\frac{d_{b_{i}}-d_{b_{i}}^{\prime}}{3}\right)+\left(t_{i}+\frac{d_{t_{i}}-d_{t_{t}^{\prime}}^{\prime}}{3}\right)(1-\alpha)$,

$x_{j} \geq 0, i \in M, j \in N, \alpha \in[0,1]$

where, for instance, $d_{c j}$ and $d_{c j}^{\prime}$ are the lateral margins (right and left, respectively) of the triangular fuzzy number central point $c_{j}$.

Consequently, by applying this approach to the previously defined FMILP model, we would obtain an auxiliary crisp MILP model as follows:

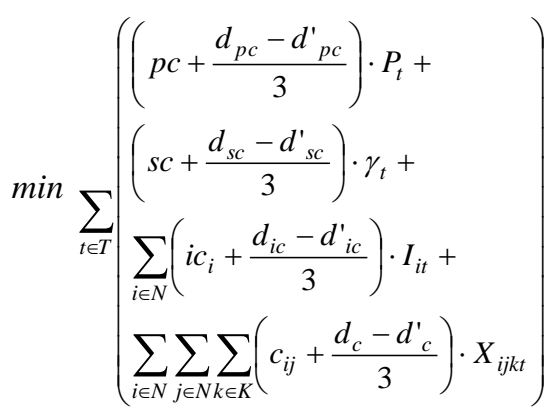

subject to:

$$
\begin{array}{r}
P_{t} \leq\left(\text { PCap }+\frac{d_{\text {PCap }}-d_{\text {PCap }}^{\prime}}{3}\right)+\left(t_{1}+\frac{d_{t_{1}}-d_{t_{1}}^{\prime}}{3}\right)(1-\alpha) \\
\forall t \in T \\
I_{i t} \leq\left(I \text { Cap }_{i}+\frac{d_{\text {ICap }}-d_{\text {ICap }}^{\prime}}{3}\right)+\left(t_{2}+\frac{d_{t_{2}}-d_{t_{2}}^{\prime}}{3}\right)(1-\alpha) \\
\forall i \in N, \forall t \in T
\end{array}
$$

and the non fuzzy constraints (2)-(4) and (7)-(14).

In order to solve the problem and according to Eq. (18), $\alpha$ is settled parametrically ( $\alpha \in[0,1])$ to obtain the value of the objective function for the different levels ( $\alpha$-cuts) of the fuzzy parameters considered in the model. The result is a fuzzy set and the planner has to decide which pair $(\alpha, z)$ is more adequate to obtain a crisp solution

\section{Case study}

In this section, we validate the proposed model as a tool for making integrated production and routing planning decisions under uncertainty.

\subsection{Implementation and resolution}

The proposed model has been developed with the modelling language MPL, and has been solved by Gurobi solver. The experiments were run in an Intel Xeon 2.93 GHz with 48 GB RAM. The model has been executed for a 6-day time planning horizon. The input data has been obtained from a bakery company located at Ibi (Alicante-Spain). This firm elaborates a special kind of frozen pre-baked bread and distributes it to a set of six customers located in several areas from Alcoy city (Al-
icante-Spain). These costumers have capacitated warehouses to store the frozen bread. Moreover, the production capacity of the manufacturing plant is also limitated. On the other hand, production, setup, inventory and transportation costs proportional to the travelled distance are also considered. Fuzzy production and inventory capacities, deterministic vehicle capacities and fuzzy costs values are shown in Table 1. Due to space limitations, the details of demand, inventory costs and transport costs are not presented here, but can be made available upon request.

\begin{tabular}{|c|c|c|c|}
\hline & rPCap & PCap & RPCap \\
\hline $\begin{array}{c}\text { Production } \\
\text { capacity }\end{array}$ & 1875 & 2250 & 2625 \\
\hline $\begin{array}{c}|c| \\
\text { Inventory } \\
\text { capacity }\end{array}$ & 215 & 250 & 285 \\
\hline \multicolumn{3}{|c|}{1050} \\
\hline $\begin{array}{c}\text { Vehicle } \\
\text { capacity }\end{array}$ & ICap & RICap \\
\hline & rpc & $p c$ & Rpc \\
\hline Production cost & 0.382 & 0.450 & 0.585 \\
\hline & rsc & sc & Rsc \\
\hline Setup cost & 27 & 32 & 37 \\
\hline $\begin{array}{c}\text { Inventory costs } \\
\text { at node } i\end{array}$ & ric $i$ & $i c_{i}$ & Ric \\
\hline$i=0$ & 0.782 & 0.920 & 1.058 \\
\hline$i=1$ & 0.425 & 0.500 & 0.575 \\
\hline$i=2$ & 0.782 & 0.920 & 1.058 \\
\hline$i=3$ & 0.952 & 1.120 & 1.288 \\
\hline$i=4$ & 1.326 & 1.560 & 1.794 \\
\hline$i=5$ & 0.425 & 0.500 & 0.575 \\
\hline$i=6$ & 0.374 & 0.440 & 0.506 \\
\hline
\end{tabular}

Table 1: PRP input data.

\subsection{Assumptions}

The main characteristics and assumptions considered in the computational experiment are the following:

- The decision variables $P_{t}, I_{i t}, Q_{i k t}$ are considered integer. Therefore, a MILP model is required to be solved.

- A single productive resource restricts the capacity of the production nodes.

- Triangular fuzzy numbers were defined by the decision makers involved in the planning process from the deviation percentages of the crisp value. These percentages ranged from an average from $15 \%$ to $25 \%$, depending on the parameter to be evaluated.

- A maximum violation of $5 \%$ is contemplated on the right-hand side of fuzzy constraints.

- Stop criteria for Gurobi solver are set to $0.5 \%$ of gap and $300 \mathrm{CPU}$ seconds.

\subsection{Evaluation of results}

This section compares the results obtained by the fuzzy PRP model with a deterministic version. The aim is to identify the possible improvements that can provide the 
consideration of uncertainty in PRP models by using fuzzy mathematical approaches.

Table 2 shows the computational efficiency of the deterministic model and the fuzzy PRP planning model proposed for this case study. The data are related to the iterations, number of constraints, variables, integers, non-zero elements, calculation time and the average density of the array of constraints. The deterministic model obtains a higher value for iterations than the fuzzy model, while the rest of parameters are identical, including the CPU time because both models arrive to the CPU time limit.

\begin{tabular}{|c|c|c|}
\hline & Deterministic & Fuzzy \\
\hline Iterations & $1,365,728$ & $1,318,611$ \\
\hline Constraints & 2609 & 2609 \\
\hline Variables & 1457 & 1457 \\
\hline Integers & 1104 & 1104 \\
\hline Non zero elements & 21,266 & 21,266 \\
\hline Array density (\%) & $0.6 \%$ & $0.6 \%$ \\
\hline CPU time (seconds) & 300 & 300 \\
\hline
\end{tabular}

Table 2: Efficiency of the Computational experiments

Table 3 summarizes the evaluation results with the different $\alpha$ values:

\begin{tabular}{|c|c|c|c|c|c|}
\hline$\alpha$ & $\begin{array}{l}\tilde{0} \\
\stackrel{0}{0} \\
\tilde{0} \\
0 \\
0 \\
0 \\
0\end{array}$ & 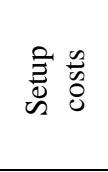 & $\begin{array}{l}\overrightarrow{0} \\
\stackrel{0}{0} \\
\stackrel{0}{0} \\
\stackrel{0}{0}\end{array}$ & 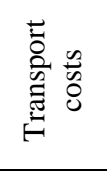 & 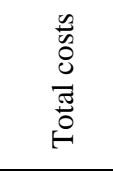 \\
\hline 0.1 & 5378.40 & 192.00 & 428.80 & 132.42 & 6131.62 \\
\hline 0.2 & 5378.40 & 192.00 & 451.68 & 132.30 & 6154.38 \\
\hline 0.3 & 5378.40 & 192.00 & 468.00 & 132.14 & 6170.54 \\
\hline 0.4 & 5378.40 & 192.00 & 484.28 & 132.14 & 6186.82 \\
\hline 0.5 & 5378.40 & 192.00 & 500.56 & 132.14 & 6203.10 \\
\hline 0.6 & 5378.40 & 192.00 & 516.84 & 132.14 & 6219.38 \\
\hline 0.7 & 5378.40 & 192.00 & 535.80 & 132.14 & 6238.34 \\
\hline 0.8 & 5378.40 & 192.00 & 552.08 & 132.14 & 6254.62 \\
\hline 0.9 & 5378.40 & 192.00 & 569.20 & 130.89 & 6270.49 \\
\hline Deterministic & 5378.40 & 192.00 & 584.64 & 130.89 & 6285.93 \\
\hline
\end{tabular}

As shown in Table 3, production and setup costs remain equal for different values of $\alpha$. Production and setup costs are strictly necessary to meet customers demand. The available production capacity makes that a setup must be done in the beginning of each period and, therefore, setup costs are the same for the deterministic model and fuzzy models with different values of the parameter $\alpha$. Moreover, it is only manufactured the exact product amounts demanded at customers locations, therefore, production costs are also identical for deterministic and fuzzy models because the demand is considered crisp. On the other hand, inventory costs present better values in fuzzy models than in the deterministic model, especially, in those models with lower values of the $\alpha$ parameter. These lower inventories imply that it could be necessary to ship higher amounts of products from the production plant to customers, increasing transport costs. In general, as seen in Table 3, all the fuzzy models, obtain better results than the deterministic model in terms of total costs. Also, those models whose $\alpha$ values come close to 1 obtain similar results to the deterministic model. This situation is logical because the closer the $\alpha$ value comes to 1 , the more similar the triangular fuzzy number model will be to a deterministic model [29].

\section{Conclusions}

This paper has proposed a fuzzy mathematical programming model for integrated production and routing planning decisions under uncertainty. The proposed PRP model considers flexible constraints as well as the lack of knowledge in objective function costs and production and inventory capacities and has been validated in a real case study corresponding to a bakery firm. The advantages of this proposal are related to: (1) the management in real situations where the information is not fully available for production and routing planning; and (2) better results in terms of total costs and similar CPU times obtained by fuzzy models with respect to the deterministic model.

Regarding to the limitations of this proposal, we have described them through further research proposals: (1) Extension of this case study in industrial environments with multiple products, more customers and a larger planning horizon; (2) consideration of travel times between nodes, waiting, loading and unloading times and sustainability issues such as fuel consumption and pollutants emissions; and (3) to develop solution procedures based on metaheuristics, bio inspired algorithms and evolutionary computation in order to solve the fuzzy PRP for large size instances in a reasonable CPU time.

\section{Acknowledgements}

This paper has been funded by the Spanish Ministry Project entitled Design and Management of Global Supply Chains (GLOBOP) (Ref. DPI2012-38061-C0201).

\section{References}

[1] B. Karimi, S. M. T. Fatemi Ghomi, and J. M Wilson, "The capacitated lot sizing problem: a review of models and algorithms," Omega, vol. 31, no. 5, pp. 365-378, 2003.

[2] N. Brahimi, S. Dauzere-Peres, N. M. Najid, and A. Nordli, "Single item lot sizing problems," Eur. J. Oper. Res., vol. 168, no. 1, pp. 1-16, 2006.

[3] R. Jans and Z. Degraeve, "Modeling industrial lot sizing problems: A review," Int. J. Prod. Res., vol. 46, no. 6, pp. 1619-1643, 2008.

[4] B. Eksioglu, A. V Vural, and A. Reisman, "The vehicle routing problem: A taxonomic review," Comput. Ind. Eng., vol. 57, no. 4, pp. 1472-1483, 2009. 
[5] G. Laporte, "The vehicle routing problem: An overview of exact and approximate algorithms," Eur. J. Oper. Res., vol. 59, no. 3, pp. 345-358, 1992.

[6] C. J. Vidal and M. Goetschalckx, "Strategic production-distribution models: A critical review with emphasis on global supply chain models," Eur. J. Oper. Res., vol. 98, no. 1, pp. 1-18, 1997.

[7] B. Bilgen and I. Ozkarahan, "Strategic tactical and operational production-distribution models: a review," International Journal of Technology Management, vol. 28. p. 151, 2004.

[8] J. Mula, D. Peidro, M. Díaz-Madroñero, and E. Vicens, "Mathematical programming models for supply chain production and transport planning," Eur. J. Oper. Res., vol. 204, no. 3, pp. 377-390, 2010.

[9] B. Fahimnia, R. Z. Farahani, R. Marian, and L. Luong, "A review and critique on integrated production-distribution planning models and techniques," J. Manuf. Syst., vol. 32, no. 1, pp. 119, 2013.

[10] J. J. Bravo and C. J. Vidal, "Freight transportation function in supply chain optimization models: A critical review of recent trends," Expert Syst. Appl., vol. 40, no. 17, pp. 6742-6757, 2013.

[11] M. Ruokokoski, O. Solyali, J.-F. Cordeau, R. Jans, and H. Süral, Efficient formulations and a branchand-cut algorithm for a production-routing problem. Groupe d'études et de recherche en analyse des décisions, 2010.

[12] Y. Adulyasak, J.-F. Cordeau, and R. Jans, "Optimization-Based Adaptive Large Neighborhood Search for the Production Routing Problem," Transp. Sci., vol. 48, no. 1, pp. 20-45, Oct. 2014.

[13] J. F. Bard and N. Nananukul, "A branch-and-price algorithm for an integrated production and inventory routing problem," Comput. Oper. Res., vol. 37, no. 12, pp. 2202-2217, 2010.

[14] P. Chandra and M. L. Fisher, "Coordination of production and distribution planning," European Journal of Operational Research, vol. 72, no. 3. pp. 503-517, 1994.

[15] F. Fumero and C. Vercellis, "Synchronized development of production, inventory, and distribution schedules," Transp. Sci., vol. 33, no. 3, pp. 330-340, 1999.

[16] L. Lei, S. Liu, A. Ruszczynski, and S. Park, “On the integrated production, inventory, and distribution routing problem," IIE Trans. (Institute Ind. Eng., vol. 38, no. 11, pp. 955-970, 2006.

[17] M. Boudia, M. A. O. Louly, and C. Prins, "Fast heuristics for a combined production planning and vehicle routing problem," Prod. Plan. Control, vol. 19, no. 2, pp. 85-96, 2008.

[18] M. Boudia and C. Prins, "A memetic algorithm with dynamic population management for an integrated production-distribution problem," Eur. J. Oper. Res., vol. 195, no. 3, pp. 703-715, 2009.

[19] M. Boudia, M. A. O. Louly, and C. Prins, "A reactive GRASP and path relinking for a combined production-distribution problem," Comput. Oper. Res., vol. 34, no. 11, pp. 3402-3419, 2007.

[20] J. F. Bard and N. Nananukul, "The integrated production-inventory-distribution-routing problem," J. Sched., vol. 12, no. 3, pp. 257-280, 2009.

[21] V. A. Armentano, A. L. Shiguemoto, and A. Løkketangen, "Tabu search with path relinking for an integrated productiondistribution problem," Comput. Oper. Res., vol. 38, no. 8, pp. 1199-1209, 2011.

[22] A. L. Shiguemoto and V. A. Armentano, "A tabu search procedure for coordinating production, inventory and distribution routing problems," Int. Trans. Oper. Res., vol. 17, no. 2, pp. 179-195, Nov. 2010.

[23] Y. Adulyasak, J.-F. Cordeau, and R. Jans, "Optimization-Based Adaptive Large Neighborhood Search for the Production Routing Problem," Transp. Sci., vol. 48, no. 1, pp. 20-45, Feb. 2014

[24] J. Mula, R. Poler, J. P. Garcia-Sabater, and F. C. Lario, "Models for production planning under uncertainty: A review," Int. J. Prod. Econ., vol. 103, no. 1, pp. 271-285, 2006.

[25] D. Peidro, J. Mula, R. Poler, and F.-C. Lario, "Quantitative models for supply chain planning under uncertainty: a review," Int. J. Adv. Manuf. Technol., vol. 43, no. 3-4, pp. 400-420, 2009.

[26] M. Díaz-Madroñero, J. Mula, and M. Jiménez, "Fuzzy goal programming for material requirements planning under uncertainty and integrity conditions," Int. J. Prod. Res., vol. 52, no. 23, pp. 6971-6988, May 2014.

[27] Y. Adulyasak, J.-F. Cordeau, and R. Jans, “The production routing problem: A review of formulations and solution algorithms," Comput. Oper. Res., vol. 55, pp. 141-152, Mar. 2015.

[28] P. Toth and D. Vigo, The vehicle routing problem, vol. 9, no. 2. Society for Industrial and Applied Mathematics, 2002.

[29] D. Peidro, J. Mula, R. Poler, and J.-L. Verdegay, "Fuzzy optimization for supply chain planning under supply, demand and process uncertainties," Fuzzy Sets Syst., vol. 160, no. 18, pp. 2640-2657, 2009.

[30] J. M. Cadenas and J. L. Verdegay, "Using fuzzy numbers in linear programming," Syst. Man, Cybern. Part B Cybern. IEEE Trans., vol. 27, pp. 1016-1022, 1997.

[31] C. V. Negoita and D. A. Ralescu, "Applications of fuzzy sets to systems analysis," 1975.

[32] J. M. Cadenas, "Design and implementation of an interactive system to solve fuzzy optimizations problems," Universidad de Granada, 1993.

[33] R. R. Y. R. R. Yager, "Ranking fuzzy subsets over the unit interval," 1978 IEEE Conf. Decis. Control Incl. 17th Symp. Adapt. Process., vol. 17, 1978.

[34] R. R. Yager, "A procedure for ordering fuzzy subsets of the unit interval," Inf. Sci. (Ny)., vol. 24, no. 2, pp. 143-161, Jul. 1981. 